\title{
N-3 polyunsaturated fatty acids induce granulopoiesis and early monocyte polarization in the bone marrow of a tMCAO rat model
}

\author{
Emőke Horváth ${ }^{1}$, Adina Huțanu ${ }^{2 *}$, Alex Orădan ${ }^{3}$, Liviu Chiriac ${ }^{4}$, Daniela Lucia \\ Muntean $^{5}$, Előd-Ernő Nagy ${ }^{6}$, Minodora Dobreanu ${ }^{7}$
}

1. Department of Pathology, University of Medicine, Pharmacy, Sciences and Technology of Târgu Mureș, Romania

2. Center for Advanced Medical and Pharmaceutical Research, University of Medicine, Pharmacy,

Sciences and Technology of Târgu Mureș, Romania

3. Center for Experimental Medicine, Iuliu Hațieganu University of Medicine and Pharmacy ClujNapoca, Romania

4. National Magnetic Resonance Center, Faculty of Physics, "Babes-Bolyai" University Cluj-Napoca, Romania. Department of Molecular Sciences, Faculty of Medicine, Iuliu Hațieganu University of Medicine and Pharmacy Cluj-Napoca, Romania.

5. Department of Analytical Chemistry and Drug Analysis, University of Medicine, Pharmacy, Sciences and Technology of Târgu Mureș, Romania

6. Department of Biochemistry, University of Medicine, Pharmacy, Sciences and Technology of Târgu Mureș, Romania

7. Department of Laboratory Medicine, Center for Advanced Medical and Pharmaceutical Research, University of Medicine, Pharmacy, Sciences and Technology of Târgu Mureș, Romania

\begin{abstract}
Introduction: Experimental acute cerebral ischemia quickly triggers circulating inflammatory cells, provoking infiltration of neutrophils and macrophages in the damaged brain region. $N-3$ polyunsaturated fatty acids alleviate the ischemic deterioration, however, their potential effect on bone marrow cell mobilization is less known.

Materials and methods: healthy male Wistar rats were submitted to intraperitoneal saline injection ( $n=10$, sham Group), transient middle cerebral artery occlusion ( $t M C A O)$ and saline injection ( $n=10$, placebo Group), tMCAO and highly purified fish-oil administration (n=10, T Group). At the two latter groups, twenty-four hours after tMCAO, MRI scans were performed to identify the ischemic regions; the eligible animals were sacrificed, the left parietal bones being removed and subjected to qualitative and quantitative histological and immunohistochemical analysis.

Results: The active hematopoietic surface was maximal at the T-Group, being significantly lower in the P-and S-Groups ( $p=0.006$ and $p=0.017$ ). The MPO positive surface increased significantly in the T-compared to the

*Corresponding author: Adina Huțanu, Center for Advanced Medical and Pharmaceutical Research, University of Medicine, Pharmacy, Sciences and Technology of Târgu Mureș, Romania. E-mail: adina.hutanu@umftgm.ro; adina_hutanu03@yahoo.com
\end{abstract}




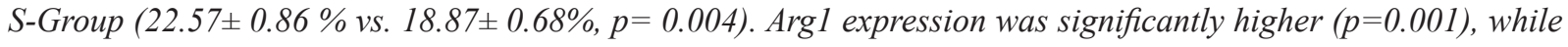
iNOS expression was lower $(p=0.004)$ in the T- than in the P-Group, but similar to the S-group. The Arg1/iNOS2 ratio was higher in the FO-treated than in the P-group $(p<0.001)$.

Conclusion: the ischemic conditions triggered granulopoiesis and the increase of iNOS2 positive, type M1 macrophage in the rat bone marrow. Fish-oil treatment generated the expansion of overall hematopoietic surface along with normalization of iNOS2, up-regulating the potentially protective Arg1 positive M2 type macrophages and causing a significant shift in the M2/M1 ratio.

Keywords: $n$-3 polyunsaturated fatty acids, hematopoiesis, M1/M2 monocytes/macrophages, digital morphometry

Received: $2^{\text {nd }}$ October 2018; Accepted: $28^{\text {st }}$ November 2018; Published: $16^{\text {th }}$ December 2018

\section{Introduction}

It is well accepted that under different pathological conditions affecting the central nervous system (neuroinflammation, stroke, autoimmune disorders, psychiatric diseases), granulocytes, monocytes and macrophages are recruited from the peripheral blood into the brain, where they cooperate with microglia in the consequent immune response (1-3). In ischemic stroke (a neurological disease, which despite optimized recanalization therapy, is one of the leading causes of death and long-term disability worldwide) (4), the early post-ischemic neuroinflammation is coordinated by polymorphonuclear neutrophil granulocytes (PMNs). These bone marrow-derived peripheral cells together with monocytes/ macrophages contribute to the breakdown of the blood-brain barrier, activation of microglia and expression of pro-inflammatory cytokines, predisposing the brain to secondary injury and poor neurological recovery (5).

There is an increasing evidence from animal models of ischemic stroke, that PMNs are accumulated in cerebral capillaries as early as after 30 minutes following middle cerebral artery occlusion (MCAO), in the first 24 hours they are also present in the ischemic core and penumbra zone (6-9). Concerning the numbers of circulating monocytes and macrophages in the cerebral capillaries and venules, these grow already at 4-6 hours post-stroke, but their massive extrav- asation into the injured parenchyma is delayed (after 3 days) (10). Depending on the microenvironmental triggers, monocytes/macrophages and microglia have a high plasticity and they can quickly transform into a different functional phenotype (11). In post-stroke immune response, the "classically-activated" pro-inflammatory macrophage phenotype (M1) amplifies the neurodegeneration in contrast to the "alternatively-activated" anti-inflammatory macrophages phenotype (M2), which has neuroprotective properties $(12,13)$. In this context, the fine-tuning of monocyte/macrophage polarization at different stages of ischemic stroke may be a therapeutical target in the future (14).

The bone marrow, along with the spleen is an important source of a wide variety of inflammatory cells, that infiltrate the cerebral tissue in acute transient ischemia. Ischemic stroke stimulates the sympathetic nervous system, activating the hematopoietic stem cells and contributes to the export of monocytes and neutrophils from bone marrow $(15,16)$, with double effect, contributing to the brain recovery but also directly aggravating the reperfusion injury (17). In this context, blocking inflammation using anti-inflammatory drugs for prevention and treatment may not be an optimal therapeutic approach. For this reason, much of the research has focused on prevention. Protective effects have been observed in animal models with neuroinflammation using a novel class of molecules produced from two omega-3 
polyunsaturated fatty acids (PUFA): docosahexaenoic acid (DHA), and eicosapentaenoic acid (EPA), collectively referred to as specialized pro-resolving mediators (SPM). Animal studies that tested these molecules in a stroke model suggested anti-neuroinflammatory properties of these n-3 PUFA via active resolution mechanisms. However, the results show significant differences, which appear due to the heterogeneity between disease models (depending on the type of n-3 PUFA, the route of administration, the dose and the duration of administration).

In the present experiment we studied the variations in polymorphonuclear neutrophils and myeloid monocyte/macrophage numbers and their phenotype in the bone marrow of rats submitted to tMCAO using a quantitative morphometric approach. In such context, it is of special interest whether fish oil (FO) containing n-3 PUFA exerts protective effects at histological level and whether the hematopoiesis is modulated or not by FO administration. We hypothesized, that the granulopoiesis and monocytes/macrophages count are influenced differently by acute ischemia in specimens with and without FO protection. We conducted detailed histomorphological and immunohistochemical analysis in order to describe and quantify the bone marrow tissue alterations in rats with tMCAO.

\section{Materials and methods}

In this study, a total of 30 mature healthy adult Wistar male rats (about 280-330 g body weight) were used, purchased from the Animal Facility, University of Medicine and Pharmacy Cluj-Napoca (Romania). Approval of the Ethics Committee of the University of Medicine and Pharmacy of Tîrgu Mureș was registered with the number 17/17.03.2016, being carried out in accordance with the Directive 2010/63/EU of the European Parliament and of the Council of 22 September 2010.

\section{The experimental protocol}

Rats were acclimated for 7 days before all experimental procedures, housed (5 animals/cage) in climate-controlled conditions (constant temperature $21-23^{\circ} \mathrm{C}$ and $12: 12$ hours light/dark cycle), standard rat chow and water being assured ad libitum. After the accommodation period, the animals were divided by a simple randomisation into three groups, each with 10 subjects: a sham group (S), a saline-treated/placebo-group (P), and a highly purified fish-oil phospholipid emulsion preconditioned group (T). The rats included in the $\mathrm{T}$ group had received a phospholipid emulsion intraperitoneally (IP) daily for 21 days, administered in a dose of $3.5 \mathrm{ml} / \mathrm{kg}$ /day, corresponding to a concentration of $70 \mathrm{mg} / \mathrm{kg}$ eicosapentaenoic acid (EPA) and $80 \mathrm{mg} / \mathrm{kg}$ docosahexaenoic acid (DHA). In the case of the animals included in the S and P groups, only $0.9 \%$ sodium chloride was administered in the same volume and route. The ischemia/reperfusion injury model was established by intraluminal filament technique: in $\mathrm{P}$ and $\mathrm{T}$ groups, ischemic stroke was induced by 90 minutes of transient middle cerebral artery occlusion (tMCAO).

Twenty-four hours after tMCAO, MRI scans were performed to identify the ischemic regions, as a control of the procedure. The S group, served as control, being subjected to similar anaesthesia and surgery with no filament insertion. The surgical procedure, anaesthesia, preoperative and postoperative monitoring conditions were performed as previously reported (18). Two animals from the placebo group $\left(\mathrm{P}_{1}\right.$ and $\left.\mathrm{P}_{4}\right)$ died shortly during the post-ischemic/reperfusion period, one animal from group $\mathrm{P}\left(\mathrm{P}_{3}\right)$ had a negligible ischemic volume on MRI examination, consequently they were excluded from the histological study. 24 hours after the ischemia, all 27 eligible animals were sacrificed under deep anaesthesia. For the histological study, the brains, spleens and left parietal bones were removed, washed in physiological saline and fixed for $24 \mathrm{~h}$ in $10 \%$ 
neutral buffered formalin for further processing. Analysing the PUFA effect in ischemia/reperfusion induced condition in $\mathrm{P}, \mathrm{T}$ and $\mathrm{S}$ groups, we focused on characterization and quantification of the active hematopoietic tissue and its cellular components, especially PMNs and myeloid monocytes/macrophages.

\section{Bone marrow examination: histological exam- ination, immunostaining and imaging}

Bone fragments were decalcified for 48 hours in $1 \%$ EDTA, processed for routine histopathology and serial sections were performed $(4-5 \mu \mathrm{m})$. One section was stained with H\&E to assess the bone marrow morphology. For immunohistochemistry (IHC), additional sections were incubated with DAKO EnVision FLEX Peroxidase-Blocking Reagent for 15 minutes. The antigen retrieval was performed in citrate solution (pH 6) using a microwave oven. After successive washing with distilled water and Tris-buffered saline ( $\mathrm{pH} 7.6)$, the sections were incubated with the following primary antibodies: anti-myeloperoxidase (MPO) antibody, clone NCL-MYELO, dilution 1:100 (Novocastra Laboratories), anti-inducible nitric oxide synthase (iNOS2), clone AA1-50, dilution 1:100 (Bioss Antibodies), polyclonal anti-Arginase, Liver (Arg1), dilution 1:200 (Bioss Antibodies) for 24 hours in a wet chamber at 4 ${ }^{0}$ C. As a secondary antibody, we used EnVision тм FLEX/HRP (Dako) in combination with a 3,3'-diaminobenzidine (DAB) solution for the chromogenic identification of the above-mentioned antigens. The conserved structure lymph nodes were used as positive controls for Arg1 and iNOS2. For MPO, the examined bone marrows (BOMs) served as a positive endogenous control of the reaction. For negative control, the primary antibodies were substituted with normal serum. Nuclei were visualised by hematoxylin counterstaining. All the morphological analyses were performed by a pathologist. Both the H\&E and immunohistochemistry slides have been scanned digitally at $20 \mathrm{X}$ magnification with Mirax Scanner, being examined with Panoramic Viewer 1.15.4 (3DHISTECH Ltd., Budapest, Hungary) through $20 \mathrm{X}$ magnification. To quantify the rat bone marrow hematopoietic subpopulations, three representative foci were chosen from complete medullary spaces from each case (without trabecular bone), they were captured with a 20 objective and saved as TIFF files. The bone marrow cellularity, as tissue surface area (H\&E stain), MPO, iNOS2, Arg1 positive cell count and total positive surface area (percent of DAB immunolabeled cells of different hematopoietic subpopulations) were measured with the image analysis software Image J $1.46(\mathrm{Na}-$ tional Institutes of Health, Bethesda, MD USA) (Ferreira and Rasband, 2012) using HSB (hue, saturation, brightness) colour filtering, a method described earlier (19). The total number of analysed images was 324, derived from the 108 immunolabeled and H\&E stained bone marrow samples.

\section{Statistics}

All registered data were analysed using the Kolmogorov-Smirnov and Shapiro-Wilk's test for distribution. As this showed normality for each parameter, we applied the t-test with Welch's correction for between-group comparison, the threshold level of statistical significance being established at $\mathrm{p}=0.05$.

\section{Results}

The results of this study are summarised in Table 1.

\section{$N-3$ polyunsaturated fatty acids effects on bone marrow cellularity}

The routine histopathologic examination of 27 parietal bone H\&E slides did not show any significant morphological changes in the structure of bone marrow, the architecture was preserved 
Table 1. N-3 polyunsaturated fatty acids effects on bone marrow cellularity, granulocytic lineage and monocyte polarisation

\begin{tabular}{lccc}
\hline & $\begin{array}{c}\text { Sham } \\
(\mathrm{S}, \mathrm{n}=10)\end{array}$ & $\begin{array}{c}\text { Placebo-treated } \\
(\mathrm{P}, \mathrm{n}=7)\end{array}$ & $\begin{array}{c}\text { PUFA-treated } \\
(\mathrm{T}, \mathrm{n}=10)\end{array}$ \\
\hline Active hematopoietic surface (HPS) & $48.46 \pm 1.38^{*}$ & $47.53 \pm 1.25^{\mathrm{H}}$ & $54.13 \pm 1.65^{*, \mathrm{H}}$ \\
\hline Myeloperoxidase (MPO) & $18.87 \pm 0.68^{* *}$ & $20.61 \pm 0.54$ & $22.57 \pm 0.86^{* *}$ \\
\hline MPO/HPS ratio & $0.39 \pm 0.02$ & $0.44 \pm 0.02$ & $0.42 \pm 0.01$ \\
\hline $\begin{array}{l}\text { Inducible nitric oxide synthase 2 } \\
\text { (iNOS2) }\end{array}$ & $1.07 \pm 0.07$ & $1.28 \pm 0.07^{\mathrm{H}}$ & $0.96 \pm 0.05^{\mathrm{HH}}$ \\
\hline Arginase 1 (Arg1) & $1.07 \pm 0.03^{* *,}$, & $0.9 \pm 0.04^{\mathrm{HH}, \%}$ & $1.30 \pm 0.05^{* *, \mathrm{HH}}$ \\
\hline Arg1/iNOS2 ratio & $1.03 \pm 0.07^{* *,}$, & $0.72 \pm 0.07^{\mathrm{HH},}$, & $1.39 \pm 0.09^{* *, \mathrm{HH}}$ \\
\hline
\end{tabular}

Active hematopoietic surface, MPO, iNOS, Arg1 expressions showed as mean \pm SE of positive surface area of the investigated fields. * $\mathrm{p}<0.05 ; * *$, H , " $\mathrm{p}<0.01 ;{ }^{\text {"t' }} \mathrm{p}<0.001$, $\mathrm{p}$ calculated with the $\mathrm{t}$ test with Welch's correction

in all three groups. Based on tissue morphology, we also observed a cellularity according to age (Figure 1a), associated with the normal maturation of the different hematopoietic (granulocytic, erythroid and megakaryocytic) lineages. (Figure 1b). Examining the myeloid/erythroid ratio of all three groups, we observed a minimally increased value in the animals given placebo.

In our ischemic model, the mean value of active haematopoietic tissue surface (HPS) determined by automated image analysis method covers $50.32 \pm 5.26 \%$ of the stromal-parenchyma structure of flat bone haematopoiesis: it was lower in the $\mathrm{P}-(47.53 \pm 1.25)$ and the $\mathrm{S}$ group $(48.46 \pm 1.38)$ compared to the FO group (54.13 $\pm 1.65)$, with statistically significant difference between $S$ and $T(p=0.017)$, respectively $P$ and $\mathrm{T}(\mathrm{p}=0.006)$. There was no significant difference between the $\mathrm{S}$ and $\mathrm{P}$ groups cellularity $(\mathrm{p}=0.626)$ (Figure 2).

\section{N-3 polyunsaturated fatty acids effects on granulocytic lineage}

Regarding the different myeloid lineages, in the FO group, we observed a mild granulocytic proliferation without an increase in the percentage of myeloblasts or enlargement of the paratra- becular unsegmented neutrophil cell layer (Figure 1c). Since image analysis on H\&E sections failed to identify the myeloid lineage, in order to characterize granulocytopoiesis in treated and non-treated ischemic groups, we used a specific marker: MPO (Figure 1d). The image analysis on IHC showed, that the bone marrow MPO positive cells surface area was significantly lower in the $\mathrm{S}$ group than in the $\mathrm{T}$ group $(\mathrm{p}=0.004)$. There was no significant difference regarding granulocytopoiesis between the two ischemic (placebo and PUFA treated) groups $(\mathrm{p}=0.75)$. In order to clarify these results, we calculated the MPOHPS ratio in all three groups, and we found it to be the highest in the ischemic P group $(0.44 \pm$ 0.02 ), without significant difference compared to values found in $\mathrm{S}$ and $\mathrm{T}$ (Table 1).

\section{Monocyte/macrophage response to acute ischemia}

In the first $24 \mathrm{~h}$ after $\mathrm{tMCAO}$, the polarization of monocytes into M1 (Figure 1e) and M2 subtypes (Figure 1f) could be observed by analysis of the tissue sections stained with the M1 and M2 specific markers. Both the iNOS2 positive (M1) and the Arg1 positive (M2) cells were relatively few in BOM stroma, but the M1 subtype was more 


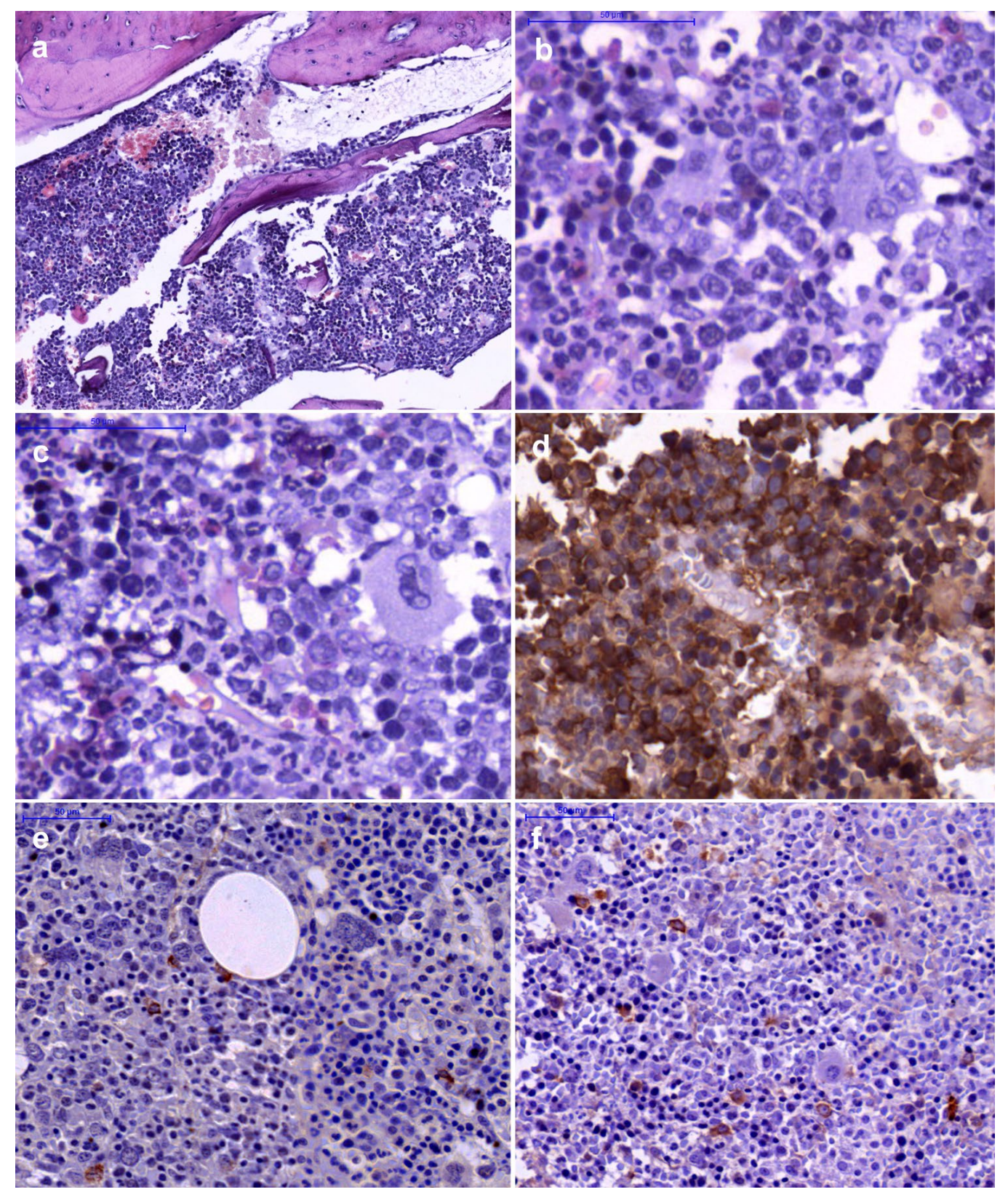

Fig. 1. Structural features of the examined bone marrows: representative images of $H \& E$ (a-c) and immunohistochemistry with MPO, iNOS2 and Arg1 (d-f). a. Hematopoietic tissue with normal cellularity. Example is shown from subject in the placebo group (3X magnification). b. At higher (20X) magnification normal maturation of different hematopoietic (granulocytic, erythroid and megakaryocytic) lineages is seen. c. The n-3 PUFA effect is characterized by mild granulocytic proliferation without an increase in the percentage of myeloblasts or enlargement of the paratrabecular unsegmented neutrophil cell layer (a subject from T group, 20X magnification).

d. Rat bone marrow MPO expression in myeloid lineage (subject from P group, 20X magnification). e-f.

Distribution of monocytes in the bone marrow stroma $24 \mathrm{~h}$ after tMCAO (DAB chromogen, 10x). Fewer iNOS2positive M1 cells (e) compared to Arg1 expressing M2 type cells (f) are present in the n-3 PUFA-treated group. 
numerous in the $\mathrm{P}$ group bone marrow (1.28 \pm $0.07)$ than in the $S(1.07 \pm 0.07)$ and $T$ groups $(0.96 \pm 0.05)$ with significant difference between $\mathrm{P}$ and $\mathrm{T}(\mathrm{p}=0.004)$. In terms of Arg1 positive surface, it was increased in the PUFA- treated $\mathrm{T}$ group $(1.30 \pm 0.05)$ compared to the control $(1.07 \pm 0.03)$ and placebo-treated groups (0.9 \pm 0.04 ), the difference being significant in both cases ( $p=0.002$ and $p=0.001$, respectively). In addition, the antigen expression in the bone marrow tissues was significantly lower in the placebo-treated group than in the sham $(\mathrm{p}=0.005)$ (Figure 1f) (Table 1). The Arg1-iNOS2 positive surface ratio in the $\mathrm{S}$ and $\mathrm{T}$ groups showed a shift in favor of the M2 cell subtype, unlike the $\mathrm{P}$ group, where the M1 phenotype was dominant (Table 1). The Arg1/iNOS2 positive surface ratio was significantly higher in the sham group than in the placebo-treated group $(\mathrm{p}=0.005)$. This ratio was elevated in the n-3 PUFA-treated group vs. the controls ( $p=0.006$ ). Given the two groups subjected to ischemia, the $\mathrm{Arg} 1 / \mathrm{iNOS} 2$ ratio was also significantly higher in the n-3 PUFA-treated $\mathrm{T}$ group than in the placebo-treated group $(\mathrm{p}<0.001)$ (Figure 3).

\section{Discussion}

It is known that ischemic stroke mobilizes hematopoietic stem cells from the bone marrow via increased sympathetic tone, leading to a myeloid dominance of hematopoiesis and an increased export of monocytes and neutrophils to the brain where they may contribute to recovery, but also to the reperfusion injury (16).

Based on our preliminary results regarding IHC analysis of the inflammatory cell populations in damaged brain tissue after tMCAO in a rat model (20), we hypothesized that acute ischemia triggered inflammatory events in the brain are associated with an increase of BOM myelopoiesis and monocyte polarization, and these chang-

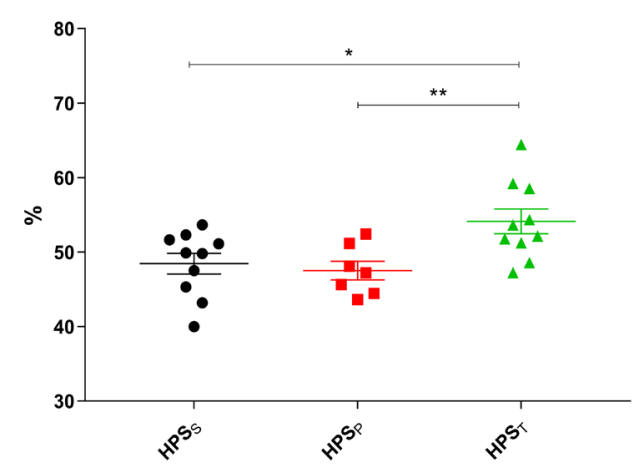

Fig. 2. Dot-plot representation of the active hematopoietic surface (HPS) in the three groups. Values showed as ratio (mean $\pm \mathrm{SEM}),{ }^{*} \mathbf{p}<0.05$, $* * \mathbf{p}<0.01$ calculated by the t-test with Welch's correction.

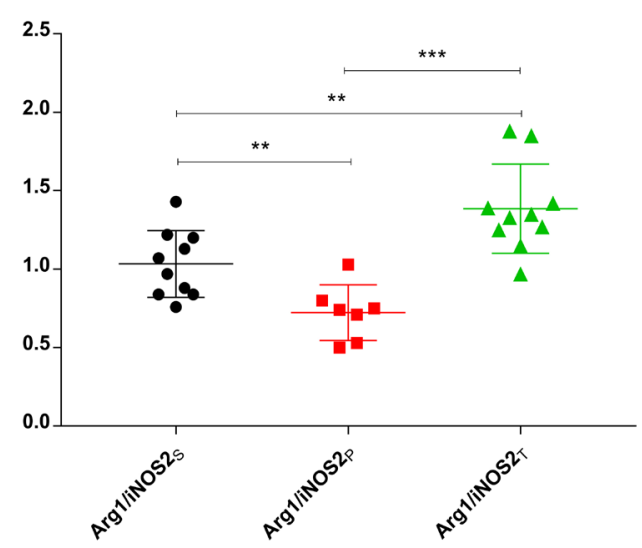

Fig. 3. Dot-plot representation of the Arg1/iNOS2 expression ratio in the three groups. Values showed as mean \pm SEM, $* * \mathbf{p}<0.01, * * * \mathbf{p}<0.001$ calculated by the t-test with Welch's correction.

es are n-3 PUFA-modulated. We compared the morphological structure of bone marrow under the influence of transient acute cerebral ischemia with and without n-3 PUFA protection in a fishoil pre-conditioned experimental rat model. $\mathrm{Hu}-$ man and rat bone marrow show a high degree of similarity in morphology, histology, and terms of hematopoietic mechanisms (21), therefore, the 
results obtained on rat should be, at least, partially translatable.

In order to detect the transient acute ischemia-induced alterations and to characterize the imbalances in hematopoietic cell populations, the classical microscopic examination of $\mathrm{H} \& \mathrm{E}$ and IHC stained rat bone marrow sections has been supplemented with automated image analysis to quantify myeloid lineage and monocytes. The feasibility of this quantitative assessment regarding the changes in bone marrow cell lineage composition was demonstrated in rodent toxicity studies (22).

In experimental stroke models, n-3 PUFA (ALA, EPA, DHA) are generally described as molecules with anti-inflammatory and pro-resolving properties decreasing infarct size and improving neurological score (3), but their effect on the two major immune organs, the spleen and the bone marrow, have not been clearly elucidated. It was demonstrated that oral feeding with polyunsaturated fatty acids fosters haematopoiesis and thrombopoiesis in healthy and bone marrow-transplanted mice, and it could serve as a possible supplemental therapy in different hematopoietic failures (23). Using complex methods for evaluating PUFA-induced changes in hematopoietic organs, Xia S and al. found that a high fish oil diet induces extramedullary hematopoiesis in the spleen, and it promotes the self-renewal of hematopoietic stem cells in the bone marrow, while a n-3 PUFA-rich fish oil diet may induce myeloid-derived suppressor cell differentiation to diminish tissue inflammation (24). In a bacteria-induced sepsis mouse model, it has been demonstrated that mice fed with a diet rich in PUFAs prior to the infection had an increased survival. Therefore, this result was attributed to the protective effect of PUFAs which stimulate granulopoiesis in the bone marrow, neutrophils having a crucial role in the early defence against bacterial infections (25).
Experimental models of transient acute cerebral ischemia describe a characteristic succesion of inflammatory events after the brain injury, associated with a strong response from the peripheral immune system and bone marrow, consisting of cytokine release (e.g. IFN- $\gamma$ ) and a robustious macrophage, neutrophil and lymphocyte efflux. Some authors found that the very first infiltrating elements are neutrophils (from 30 minutes to 12 hours), $(6,7,8)$ whereas others observed the early recruitment of blood-derived macrophages (at 12 hours) and a later appearance of neutrophils (1-3 days) (9), which is why the target of this research was the bone marrow as the major source of the above-mentioned inflammatory cells.

In our study, we found a significant expansion of the active hematopoietic tissue surface in the n-3 PUFA treated group in comparison with the control and placebo groups. Using image analysis for the quantification of the MPO positive cells, appreciated as positive surface area (expressed as percentage of DAB immunolabelled MPO positive cells), the granulocytopoiesis was more pronounced in the PUFA preconditioned ischemic group than in the control group, which itself indicates that PUFA enhances the granulocytopoiesis. However, by examining the MPO positive surface/active hematopoietic surface ratios in all three groups, we assumed that (although no significance can be observed) the granulocytopoiesis is primarily stimulated by ischemia. The combined effect of preconditioning and ischemia resulted primarily in an increase in haematopoiesis, reducing this ratio in the $\mathrm{T}$ group.

Regarding the bone marrow monocytes/macrophages, they were under-represented in comparison to other cells, but this finding does not diminish their significance in ischemia-induced inflammation, which is at least equivalent to granulocytes. Macrophage precursor cells in bone marrow differentiate into various subpopulations of monocytes, which possess distinct antigen profile $(15,26)$. Since M1 and M2 macrophages 
and microglia can be converted into each other due to environmental stimuli, their polarization might have a specific importance in the control of inflammation in stroke (27). iNOS2 and Arg1 are two functionally related, key enzymes in monocytes/macrophages, which characterize this polarization: while the former is present on the pro-inflammatory M1 cells, the latter is specific for the protective M2 subtype $(28,14)$. We investigated the presence of iNOS2-positive and Arg1-positive cells in our bone marrows, observing that iNOS2 shows a higher expression in the placebo-treated ischemic group, while the FO treatment caused a significant M2/M1-phenotype shift in the favour of Arg1. Even though iNOS2 and Arg1 positivity is mild in all bone marrow, the elevation of the potentially neuroprotective M2 type macrophages in the FO-treated group is a significant finding.

Our study has a few limitations. The results are based on immunohistochemical evaluation, assisted by semiquantitative morphometry with staining the cell types of interest on a serial section. We did not perform flow cytometry, the most quantitative method able to detect the shifts in the composition of different bone marrow cell population. The combination of the two methods would contribute to fully understand the effect of n-3 PUFAs on haematopoiesis following tMCAO.

\section{Conclusions}

According to our findings, we conclude that n-3 PUFAs influence the inflammatory response at the bone marrow level shortly after tMCAO. Our data show that in experimentally induced acute ischemic condition n-3 PUFA pretreatment enhances haematopoiesis and granulocytopoiesis. The beneficial effect of PUFAs in acute ischemic condition is also reflected by their substantial role in early monocyte polarisation: reduction of iNOS2 to levels even lower than the normal, and up-regulation of Arg1 expression. This results in an important shift in the M2/M1 type ratio in favour of the Arg1-positive, potentially protective subpopulation. This phenotype might possess importance in the targeted therapy in acute cerebral ischemia.

\section{Funding Sources}

The current research was supported by an Internal Research Grant of the University of Medicine and Pharmacy Târgu-Mures, Romania (Number 17803/1/22.12.2015) and partly funded by Studium Prospero Foundation, Romania (Number 1547/18.12.2015).

\section{Acknowledgements}

The morphological analyses used the infrastructure of the Advanced Medical and Pharmaceutical Research Centre of the University of Medicine and Pharmacy Târgu-Mureș. The authors would like to thank Genoveva Rigmányi for technical assistance.

\section{Competing financial interests}

The authors declare no competing financial interests.

\section{Author Contributions}

All authors have contributed significantly, and all authors are in agreement with the content of the manuscript.

E. Horváth: principal investigator of the project, morphological and IHC analyses, acquisition and interpretation of histological data and automated image analysis, data analysis and interpretation, manuscript writing, collection of literature data; A. Orădan: surgical procedures and tissue sample collection; L. Chiriac: $3 \mathrm{D}$ reconstruction and ischemic evaluation; $\mathbf{M}$. Dobreanu: conception and design, administrative support, final approval of manuscript; E-E. 
Nagy: statistical analysis, data interpretation, manuscript writing; D. Muntean: administrative support, final approval of manuscript; A. Huțanu: co-investigator of the project, experiment and study design, collection of literature data, data interpretation, manuscript writing.

\section{Abbreviations}

tMCAO, transient middle cerebral artery occlusion; (S), sham group; (P), saline-treated/placebo- group; (T); I/R, ischemia/reperfusion; highly purified fish-oil (FO) phospholipid emulsion preconditioning group DHA, docosahexaenoic acid; EPA, eicosapentaenoic acid; n-3 PUFAs, n-3 polyunsaturated fatty acids; MRI, magnetic resonance imaging; BOM, bone marrow; PMNs, polymorphonuclear neutrophils; H\&E, haematoxylin and eosin; IHC, immunohistochemistry; $\mathrm{DAB}, 3,3$ '-diaminobenzidine; HPS, active haematopoietic surface; M1, ,classically-activated" pro-inflammatory macrophages phenotype; M2, ,alternatively-activated" anti-inflammatory macrophages phenotype; MPO, Myeloperoxidase, iNOS2, inducible nitric oxide synthase; Arg1, arginase

\section{References}

1. Takahashi Y, Yu Z, Sakai M, Tomita H. Linking Activation of Microglia and Peripheral Monocytic Cells to the Pathophysiology of Psychiatric Disorders. Front Cell Neurosci.2016 Jun;10:144. DOI: 10.3389/fncel.2016.00144

2. Wimmer I, Zrzavy T, Lassmann H. Neuroinflammatory responses in experimental and human stroke lesions. J Neuroimmunol.2018 Oct; 323:10-18. DOI: 10.1016/j. jneuroim.2018.07.003

3. Trépanier MO, Hopperton KE, Orr SK, Bazinet RP. $\mathrm{N}-3$ polyunsaturated fatty acids in animal models with neuroinflammation: An update. Eur J Pharmacol. 2016 Aug;785:187-206. DOI: 10.1016/j.ejphar.2015.05.045

4. The World Health Organization (WHO) updates fact sheet on Top 10 causes of Death, https://communitymedicine4asses.wordpress.com/2017/02/01/who-updates-fact-sheet-on-top-10-causes-of-death-27-january-2017/

5. Hermann DM, Kleinschnitz C, Gunzer M. Implications of polymorphonuclear neutrophils for ischemic stroke and intracerebral hemorrhage: Predictive value, pathophysiological consequences and utility as therapeutic target. J Neuroimmunol. 2018 Aug;321:138-43. DOI: 10.1016/j.jneuroim.2018.04.015

6. Jin R, Yang GJ, Li GH. Inflammatory mechanisms in ischemic stroke: role of inflammatory cells. J Leukoc Biol. 2010 May;87(5):779-89. DOI: 10.1189/ jlb.1109766

7. Ceulemans A-G, Zgavc T, Kooijman R, Hachimi-Idrissi S, Sarre S, Michotte Y. The dual role of the neuroinflammatory response after ischemic stroke: modulatory effects of hypothermia. J Neuroinflammation 2010 Nov;7:74. DOI: 10.1186/1742-2094-7-74. DOI: 10.1186/1742-2094-7-74

8. Kriz J. Inflammation in ischemic brain injury: timing is important. Crit Rev Neurobiol. 2006;18 (1-2): 145-57. DOI: $10.1615 /$ CritRevNeurobiol.v18.i1-2.150. DOI: 10.1615/CritRevNeurobiol.v18.i1-2.150

9. Grönberg NV, Johansen FF, Kristiansen U, Hasseldam H. Leukocyte infiltration in experimental stroke. J Neuroinflammation 2013 Sep; 10: 115. DOI: 10.1186/17422094-10-115

10. Ritzel RM, Patel AR, Grenier JM, Crapser J, Verma R, Jellison ER, et al. Functional differences between microglia and monocytes after ischemic stroke. J Neuroinflammation. 2015 May; 12:106. DOI: 10.1186/s12974015-0329-1

11. Boche D, Perry VH, Nicoll JA. Activation patterns of microglia and their identification in the human brain. Neuropathol Appl Neurobiol. 2013 Feb;39(1):3-18. DOI: 10.1111/nan.12011

12. Ma Y, Wang J, Wang Y, Yang GY. The biphasic function of microglia in ischemic stroke. Prog Neurobiol. 2017 Oct; 157:247-72. DOI: 10.1016/j.pneurobio.2016.01.005

13. Xiong XY, Liu L, Yang QW. Functions and mechanisms of microglia/macrophages in neuroinflammation and neurogenesis after stroke. Prog Neurobiol. 2016 Jul;142:23-44. DOI: 10.1016/j.pneurobio.2016.05.001

14. Kanazawa M, Ninomiya I, Hatakeyama M, Takahashi T, Shimohata T. Microglia and monocytes/macrophages polarization reveal novel therapeutic mechanism against stroke. Int J Mol Sci. 2017 Oct;13:18(10). pii: E2135.

15. Gliem M, Schwaninger M, Jander S. Protective features of peripheral monocytes/macrophages in stroke. Biochim Biophys Acta. 2016 Mar;1862(3):329-38. DOI: 10.1016/j.bbadis.2015.11.004

16. Courties G, Herisson F, Sager HB, Heidt T, Ye Y, Wei $\mathrm{Y}$, et al. Ischemic stroke activates hematopoietic bone marrow cells. Circ Res. 2015 Jan;116(3):407-17. DOI: 10.1161/CIRCRESAHA.116.305207

17. Wolf D, Ley K. Waking up the stem cell niche: how hematopoietic stem cells generate inflammatory monocytes after stroke. Circ Res. 2015 Jan;116(3):389-92. 
DOI: 10.1161/CIRCRESAHA.114.305678

18. Orădan A, Huțanu A, Horváth E, Chiriac L, Dobreanu M. Improved rat stroke model by intraluminal middle cerebral artery occlusion: a special emphasis on surgical technique. Health Problems of Civilization 2017;11:202-10. DOI: 10.5114/hpc.2017.70008

19. Mezei T, Horváth E, Turcu M, Gurzu S, Raica M, Jung I. Microvascular density in non-Hodgkin B-cell lymphomas measured using digital morphometry. Rom J Morphol Embryol. 2012; 53(1):67-71.

20. Horváth E, Adina Hutanu A, Chiriac L, Dobreanu M, Orădan A, Nagy EE . Ischemic damage and early inflammatory infiltration are different in the core and penumbra lesions of rat brain after transient focal cerebral ischemia. J Neuroimmunol. 2018;324:35-42. DOI: 10.1016/j.jneuroim.2018.08.002

21. Romaniuc A, Lyndina Y, Sikora V, Lyndin M, Karpenko L, Gladchenko O, et al. Structural features of bone marrow. Interv Med Appl Sci 2016 Sep;8(3):121-6. DOI: $10.1556 / 1646.8 .2016 .3 .3$

22. Kozlowski C, Fullerton A, Cain G, Katavolos P, Bravo J, Tarrant JM. Proof of concept for an automated image analysis method to quantify rat bone marrow hematopoietic lineages on H\&E sections. Toxicol Pathol 2018 Apr;46(3):336-47. DOI: 10.1177/0192623318766458

23. Limbkar K, Dhenge A, Jadhav DD, Thulasiram HV, Kale V, Limaye L et al. Oral feeding with polyunsat- urated fatty acids fosters hematopoiesis and thrombopoiesis in healthy and bone marrow-transplanted mice. J Nutr Biochem. 2017 Sep;47:94-105. DOI: 10.1016/j. jnutbio.2017.05.002

24. Xia S, Li XP, Cheng L, Han MT, Zhang MM, Shao QX, et al. Fish Oil-Rich Diet Promotes Hematopoiesis and Alters Hematopoietic Niche. Endocrinology 2015 Aug;156(8):2821-30. DOI: 10.1210/en.2015-1258

25. Svan SL, Varemo L, Gabrielsson BG, Peris E, Nookaew I, Grahnemo L, et al. Six tissue transcriptomics reveals specific immune suppression in spleen by dietary polyunsaturated fatty acids. PLoS One 2016 May; 11 (5): e0155099. DOI: 10.1371/journal.pone.0155099

26. ElAli A, Jean LeBlanc N. The role of monocytes in ischemic stroke pathobiology: New avenues to explore. Front Aging Neurosci. 2016 Feb;8:29. DOI: 10.3389/ fnagi.2016.00029

27. Nakagawa Y, Chiba K. Role of microglial M1/M2 polarisation in relapse and remission of psychiatric disorders and diseases. Pharmaceuticals (Basel) 2014 Nov; 7(12): 1028-48. DOI: 10.3390/ph7121028

28. Rath M, Müller I, Kropf P, Closs EI, Munder M. Metabolism via Arginase or Nitric Oxide Synthase: Two Competing Arginine Pathways in Macrophages. Front Immunol. 2014 Oct;5:532. DOI: 10.3389/fimmu.2014.00532 